\title{
Cost-effectiveness of an insertable cardiac monitor in a high-risk population in the US
}

\author{
Mitchell Elkind ${ }^{1}$, Klaus Witte ${ }^{2}$, Scott Kasner ${ }^{3}$, Laura Sawyer ${ }^{4}$, Frank Grimsey Jones ${ }^{4}$, \\ Claudia Rinciog ${ }^{4}$, Stelios Tsintzos ${ }^{5}$, Sarah Rosemas $^{5}$, David Lanctin ${ }^{5}$, Paul Ziegler ${ }^{6}$, and \\ Matthew Reynolds ${ }^{7}$ \\ ${ }^{1}$ Columbia University Vagelos College of Physicians and Surgeons \\ ${ }^{2}$ University of Leeds \\ ${ }^{3}$ University of Pennsylvania \\ ${ }^{4}$ Symmetron Ltd \\ ${ }^{5}$ Medtronic Inc \\ ${ }^{6}$ Medtronic, Inc. \\ ${ }^{7}$ Lahey Hospital and Medical Center
}

July 29, 2021

\begin{abstract}
Objective: To evaluate the cost-effectiveness of insertable cardiac monitors (ICMs) compared to standard of care (SoC) for detecting atrial fibrillation (AF) in patients at high risk of stroke (CHADS2 >2), in the US. Background: ICMs are a clinically effective means of detecting AF in high-risk patients, prompting the initiation of non-vitamin $\mathrm{K}$ oral anticoagulants (NOACs). Their cost-effectiveness from a US clinical payer perspective is not yet known. Methods: Using patient data from the REVEAL AF trial $(n=446$, average CHADS2 score $=2.9)$, a Markov model estimated the lifetime costs and benefits of detecting AF with an ICM or with SoC (namely, intermittent use of electrocardiograms [ECGs] and 24-hour Holter monitors). Ischemic and hemorrhagic strokes, intra- and extra-cranial hemorrhages, and minor bleeds were modelled. Diagnostic and device costs were included, plus costs of treating stroke and bleeding events and of NOACs. Costs and health outcomes, measured as quality-adjusted life years (QALYs), were discounted at $3 \%$ per annum. One-way deterministic and probabilistic sensitivity analyses (PSA) were undertaken. Results: Lifetime per-patient cost for ICM was $\$ 58,132$ vs. $\$ 52,019$ for SoC. ICMs generated a total 7.75 QALYs vs. 7.59 for SoC, with 34 fewer strokes projected per 1,000 patients. The incremental cost-effectiveness ratio (ICER) was $\$ 35,452$ per QALY gained. ICMs were cost-effective in $72 \%$ of PSA simulations, using a $\$ 50,000$ per QALY threshold. Conclusions: The use of ICMs to identify AF in a high-risk population is likely to be cost-effective in the US healthcare setting.
\end{abstract}

\section{Hosted file}

US HRAF HF Manuscript_FINAL.docx available at https://authorea.com/users/428276/articles/ 532176-cost-effectiveness-of-an-insertable-cardiac-monitor-in-a-high-risk-population-inthe-us 